\title{
An Environmental Transmission Electron Microscope for In-Situ Observation of Chemical Processes at the Nanometer Level
}

\author{
Renu Sharma ${ }^{1}$, Peter A. Crozier ${ }^{1}$, Ronald Marx ${ }^{2}$ and Karl Weiss ${ }^{1}$ \\ ${ }^{1}$ Center for Solid State Science, Arizona State University, Tempe, AZ 85287-1704. \\ ${ }^{2}$ FEI, FEI Electron Optics B.V., Eindhoven - The Netherlands
}

High-resolution electron microscopy (HREM) is an important technique for understanding nanotechnology processes. In-situ observations of gas-solid reactions involving nano-particles requires a TEM capable of handling gaseous environments around the sample while maintaining high-resolution capabilities at elevated temperature. During the past decade, various research groups have designed and/or modified commercially available instruments to successfully create environmental transmission electron microscopes (ETEM). Such instruments have been successfully employed to obtain time and temperature resolved electron diffraction patterns and atomic-resolution images not only at high temperatures but also under controlled gaseous environments [1,2 and references therein]. Here we present the first commercially available digitally controlled ETEM/ESTEM capable of obtaining high-resolution images, electron diffraction patterns (EDP), electron energy-loss spectra (EELS), chemical maps (energy-filtered images) and annular dark-field Z-contrast images under reaction conditions. Such an ETEM has been rightfully described as a nanosynthesis and nano-characterization lab fitted inside the column of the microscope.

The basic microscope is a Tecnai F-20 (field emission gun) TEM/STEM and the modifications to accommodate high gas pressures in the sample area (environmental cell) are based on the principle of differential pumping. The microscope column has been modified to add three sets of differential pumping apertures for three pumping stages between the gun valve and viewing chamber. The first two sets of apertures are placed within the top and the bottom polepieces of the objective lens and some of the gas leaking through this first set of apertures is pumped out using a turbo-molecular pump (TMP). The second stage is between the condenser aperture and selected area aperture and is pumped using a molecular drag pump (MDP). An ion pump is employed for the last stage before the gun valve. There is control box to open and close various pneumatic valves and thus the microscope can be switched between ETEM and TEM mode easily. The gas in-let is controlled by a set of shut off valves and pressure is regulated using a fine needle valve. A residual gas analyzer (RGA) is used to analyze gas coming out of the reaction chamber (sample area). The gas pressures are monitored at different points in the system.

A high-resolution image of gold particles on carbon recorded using a CCD camera at the end of the Gatan Imaging Filter (GIF) (Figure 1a) in 12 mbar of $\mathrm{N}_{2}$, shows two dimensional lattice spacing of $0.235 \mathrm{~nm}$. Similarly lattice fringes in Mo catalyst particles can be clearly resolved at $760^{\circ} \mathrm{C}$. This instrument has a point-to-point resolution of $0.24 \mathrm{~nm}$ and a probe size of $0.2 \mathrm{~nm}$. More importantly, figure $1 \mathrm{a}$ and $1 \mathrm{~b}$ show the digital diffractograms of evaporated gold particles on a thin carbon film, recorded before and after the external modifications for ETEM were installed. The information limit was measured to be at $0.13 \mathrm{~nm}$ and was preserved in 4 mbar of $\mathrm{H}_{2}$ (Figure 2c). Recent results from chemical vapor deposition and oxidation/reduction experiments will be presented. 

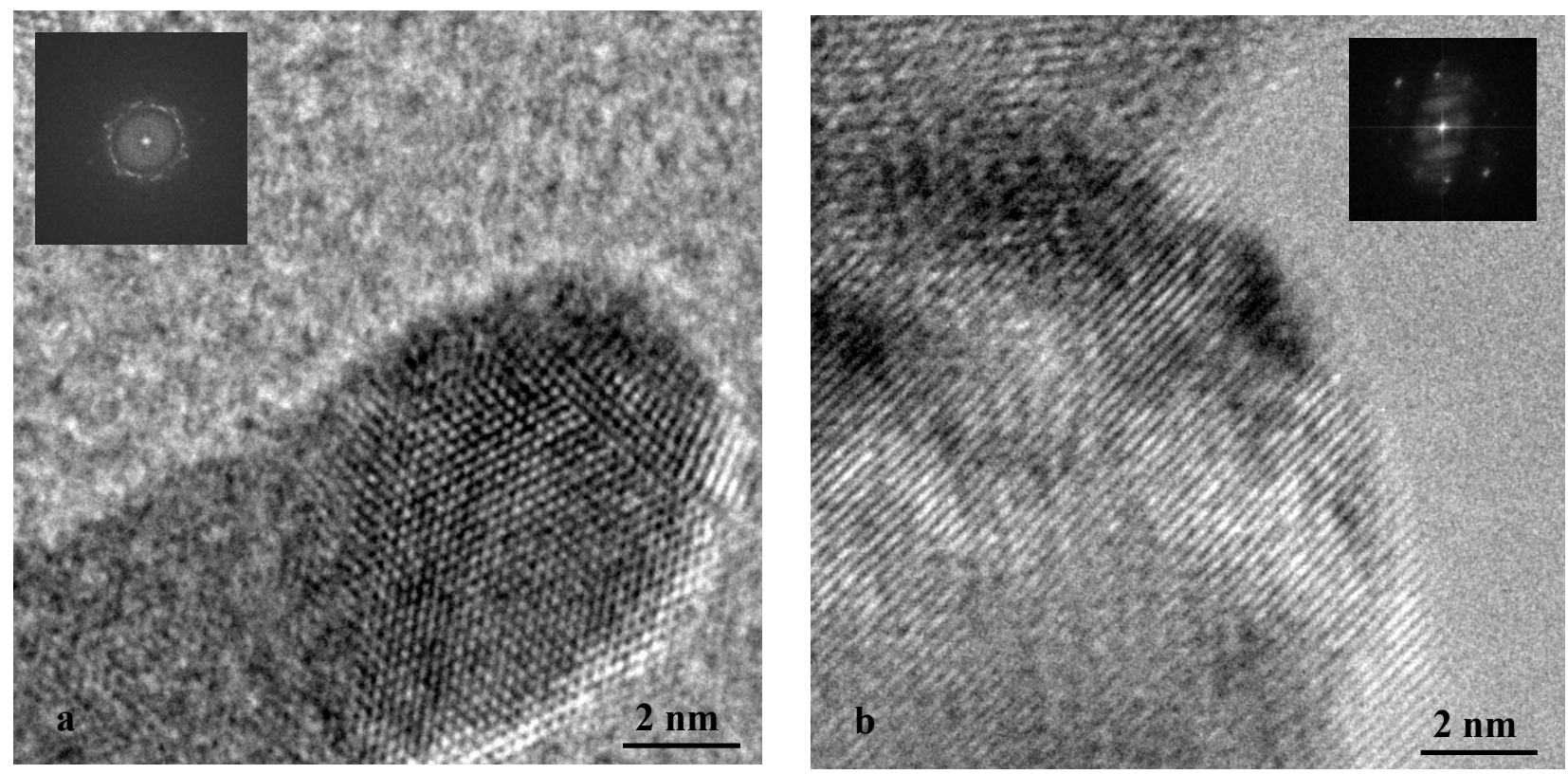

Figure1. HREM images of (a) gold particle in 12 mbar of $N_{2}$ at room temperature and (b) Mo catalyst particle at $760^{\circ} \mathrm{C}$ in vacuum recorded using a $1024 \times 1024 \mathrm{CCD}$ camera. The respective digital diffractograms are inset in the images.
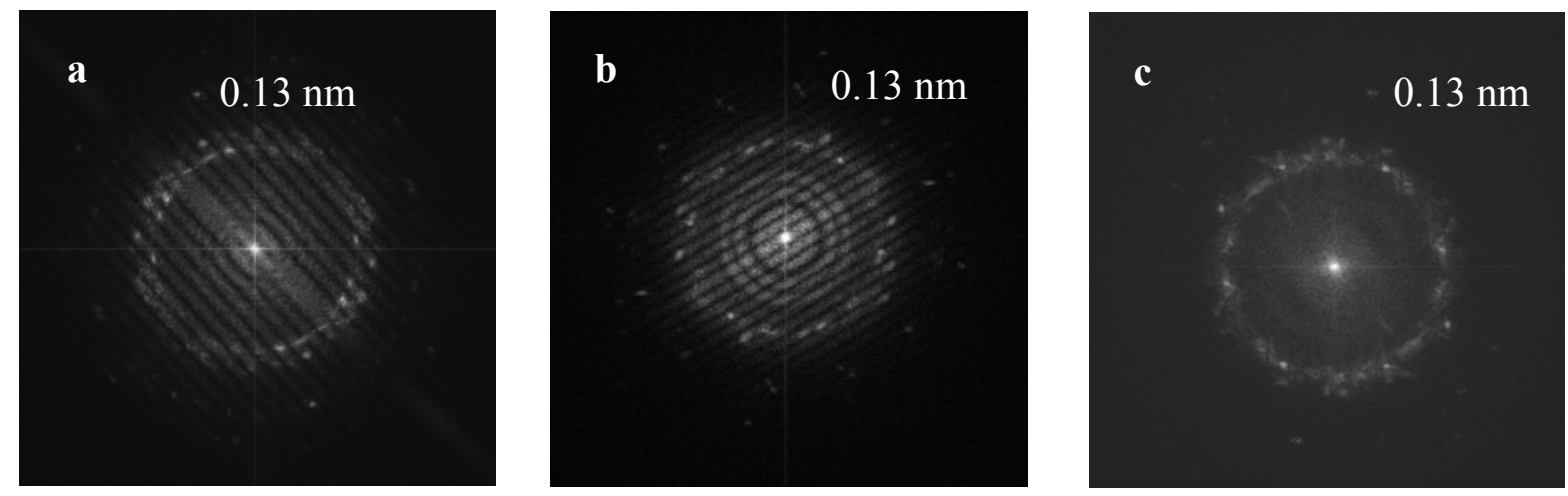

Figure 2. Digital diffractogram of HREM images showing 0.13 information limit, recorded (a) after installation of basic microscope, (b) after installation of external plumbing for environmental control in the sample region and (c) in 4 Torr of $\mathrm{H}_{2}$ gas.

\section{References:}

1. Gai,P.L. (1997) Solid Catalysis and Porous Solids, 4, (1999) 63

2. Sharma, Renu, Microscop. Microanal. , 7, (2001) 494,

3. Hansen et al, Science, 294, (2000) 1508.

4. The Center for High Resolution Electron Microscopy, Prof. David Smith, Dr. Jeff Drucker and grants from DOE (DE-AC3699GO10331) and NSF (DMR-0216715) are gratefully acknowledged. 